Session 1520

\title{
Web Programming for Industrial Applications: A New Course
}

\author{
Paul I-Hai Lin, Hal Broberg \\ Indiana University-Purdue University Fort Wayne
}

\begin{abstract}
An explanation of a new course currently being taught at the undergraduate and graduate level is provided. Topics including a survey of Internet and Web, HTML, JavaScript/JScript, Java Applet, Perl, CGI protocol and scripts, and Web-based databases are covered. Some theory in industrial process control, sensors, and transducers is introduced with emphasis on Web-based data acquisition, industrial control and automation applications. Laboratory experiments accompanying this course are outlined. Suggestions for selecting laboratory hardware and software are provided.
\end{abstract}

\section{Introduction}

There is an increasing demand from industry for professionals who understand the key issues and know how to solve the real world problems using a knowledge of networking, Web technology, and Internet-based control systems. In response to this need, the author's department offered a senior-level course on "Web Programming for Industrial Applications" for the first time in August 2000 as part of a computer networking certificate program.

With our experience in delivering synchronous TV distance learning course and asynchronous Web-based courses, we tried to deliver this course using a combination of TV lectures and hands-on lab via the Web to provide flexible time for class instruction and learning activities. The TV lecture portion (three-hour of lecture per week) also allowed students who have time conflicts to watch videotapes provided by the university. The hands-on lab via the Web (www.etcs.ipfw.edu/ lin) is a cost effective solution that enables us to offer high-technology courses with lab components without extensive resources. A series of hands-on labs were developed and hosted by a Web server for use by students using a Web browser. The lab setups can be accessed by students 24-hours a day, anywhere in the world, without coming to the university.

The rest of paper is organized as follows. Course design issues are introduced in Section II. The Web-based laboratory environment is discussed in Section III. Course evaluation and conclusion are provided in Section IV. 


\section{Course Design}

The Electrical and Computer Engineering Technology department (ECET) delivers courses to distance learners, part-time students who work full time, and students who have class time conflicts. In 1992, the department began to offer technical courses that include distance-learning section. However, all students who signed up for TV and video sections are still required to attend lab for the course. We tried a different approach for this new course and expected the following benefits:

- Greater Scheduling Flexibility: with the availability of hands-on lab via the Internet, students would be able to receive a complete technical distance course from ECET department without coming to Indiana University-Purdue University Fort Wayne (IPFW).

- Cost Effective: we recognize this delivery method is cost effective for offering technical courses with a lab component, because it requires only one set of the equipment while traditional labs require multiple sets of the same equipment.

- Reach Out to Broader Audiences: it not only assists students who need flexible schedules but also allows us to reach out to audiences that are looking for hightech courses for their professional development or continuing education.

- Future Expansion: experience of developing hands-on labs via the Web will enable us to convert the labs of many of our courses such as Introduction to Data Communications, and Introduction to Control Systems to asynchronous delivery.

The course was given three different course numbers: EET 499, CPET 499, and CE 597 ( 3 credit hour). It was delivered with a combination of the following:

- Live lecture in LB 35 TV Studio and Channel 56 Cable TV

- A lab setup (room ET 213) for Internet Access using a Lab Server: http:149.146.36.204

- Video tape distribution

- A WebCT Site: http://courses.ipfw.edu:8900/

- A course Web page: www.etcs.ipfw.edu/ lin

The course description follows: Web Programming For Industrial Applications (4cr): A survey of techniques and technologies for Internet and Web applications including World Wide Web, computer networking, TCP/IP applications, HTML (Hypertext Markup Language), JavaScript/JScript, Java Applet, Perl (Practical Extraction Report Language), CGI (Common Gateway Interface), XML (eXtensible Markup Language), Visual Basic, Visual C++, Web-based databases, etc. Basics of industrial process control, sensors, and transducers. Emphasis on Web-based data acquisition, industrial control and automation applications.

The course outline is shown below:

- Week 1,2 - Communications/Computer Networks and Services

- Week 3 - Overview of Internet Technology: Internet Web-enabled Applications (Education, Content Delivery/Advertisement, B2C, B2B, Machine Control and Monitoring, Electronic Publishing with Multimedia Technology, and Database applications); Multimedia Authoring Environment 
- Week 4 - Web Client/Server Applications: Web browser, Web pages, Web Server, and HTTP Protocol

- Week 5, 6, 7 - Programming Languages for Web-based Applications (A Survey of Web Programming Technologies, HTML, XML, JavaScript, JScript, ECMAScript, VBScript, Java Applet, and Perl); HTML Documents ( HTML structure and elements, HTML Tags and Attributes, HTML Page Authoring Tools, Web Page Creation, Tables, Forms, Frames, Creating guest books, and Industrial Application Web Pages); Dynamic Web HTML Programming Environment (JavaScript/JScript, Java Applet, CGI Scripting)

- Weeks 8, 9, 10 - Web Applications with JavaScript/Jscript (JavaScript and HTML, Execution of JavaScript programs, Form and form elements, and Debugging JavaScript, Basics of JavaScript, JavaScript objects, Using window objects and dialog boxes, Using arrays object for collections of data, Controls objects, Processing clientside form for collecting user information, Handling events, Working with images and animation, JavaScript-enriched Industrial Application Web Pages )

- Week 11, 12 - Web servers and Database (Server selection, setup, maintenance, thin Web server example, and Lab server, intro to database and interface)

- Week 13, 14 - Perl and CGI (Perl programming language, syntax, regular expressions, Perl/CGI, C/C++, and Visual Basic CGI script)

- Week 15 - Building blocks for Web-based controls and measurement system

The steps required to design and implement the course were:

1. Development of a syllabus, a lesson design for videotape lectures, and a lab design

2. Acquire software and hardware materials for lab development

3. Setup the Web server, Web camera, and Web site

4. Design programs and Web pages for; student access control (ID and password), to gather information on user log-in and connection time, to use the Web camera

5. Design, code post, and test the Web-based labs

6. Design and write Web pages for posting the syllabus, lecture materials, homework, and lab assignments

7. Use the Email system for communicating with students, receiving and returning homework assignments and lab reports

8. Develop a course evaluation plan

9. Complete summative evaluation exercises

\section{The Web-Based Laboratory Environment}

Major hardware components of the lab include a Windows98 PC (Web server: http://149.164.36.204), Opto22 SNAP B-300 Ethernet I/O Brain (10/100 Mbps, Web server), SNAP I/O Modules (digital-to-analog converters, digital-to-analog converters, and digital I/Os), Opto22 Controller (LCM4), Web Camera, a DC motor control system, a single phase AC motor, and sensors (current, voltage, temperature, humidity). The camera Web page, as shown in Figure 1, is designed to include a light ON/OFF control option so that any user can view the lab setups even when the ceiling light of the lab room is turned off. 


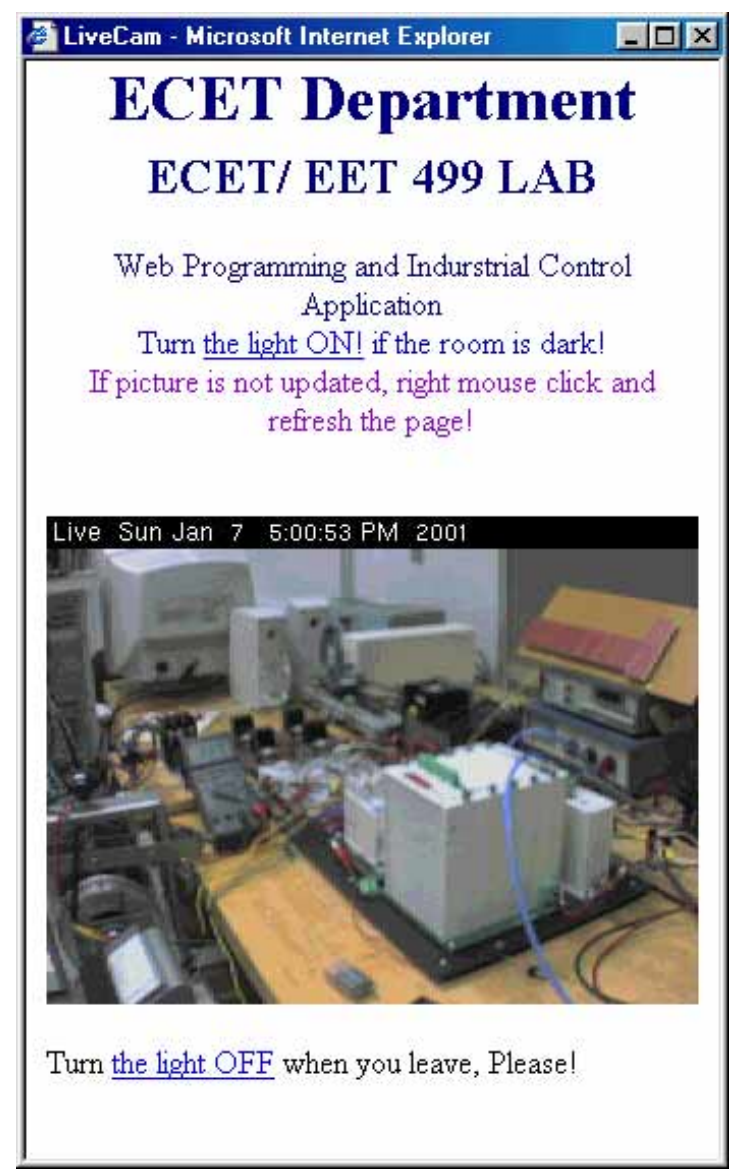

Figure 1. A Snap Shot of Web Cam Looking at the Lab Equipment

Each student is assigned a password and a user ID to use the lab. To familiarize students with the Web-based laboratory environment, the Lab server is accessible without any restriction for about 8 weeks. To avoid conflicts in accessing control system hardware, we then change the Lab server to a time-slot reservation basis. Each student can schedule the lab time (up to 4 hours) through a lab scheduling Web page. A server-side program runs in the background to record and report the access time and frequency for each authorized user. However, the Web Camera can be accessed anytime without restrictions.

Students enrolled in either the TV Channel 56 or Video Tape sections are required to use their own PC running Microsoft Windows 95/98/2000 with an Internet connection and the following software: Microsoft Visual InterDev, Visual Basic 6.0, Visual C++ 6.0. Those students who enrolled in the EET 499-01 and CPET 499-01 sections may use IPFW and/or ECET labs PCs and are not required to use their own PC.

We developed 13 lab experiments for the course, however, labs numbered 7, 8, 9, 12, and 13 were skipped due to their difficulty and excessive learning time required to prepare Visual Basic programs on the user side to run the labs. The lab Web page is shown in Figure 2. 


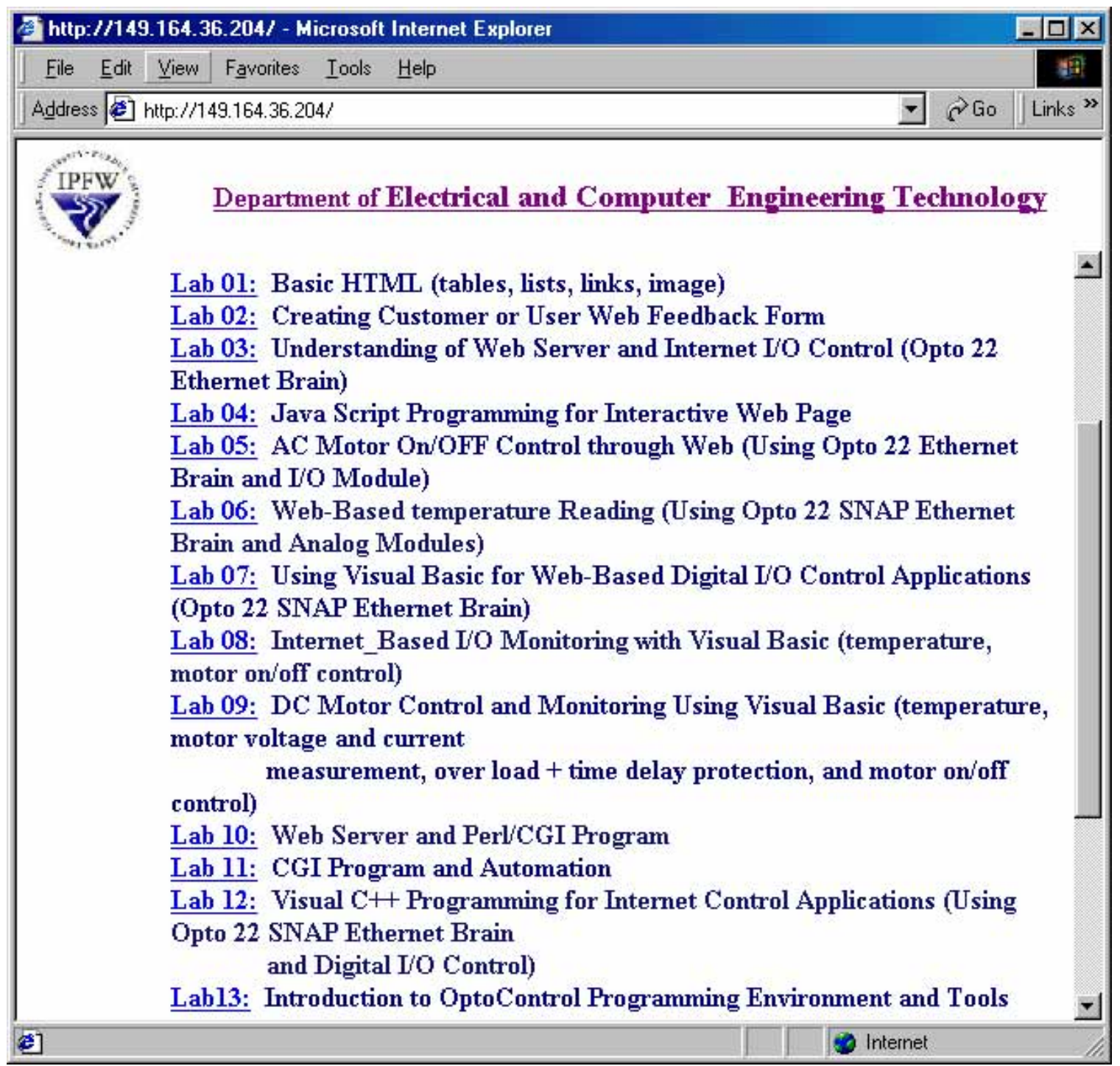

Figure 2. The Laboratory Web Page

A typical lab Web page for the course is written in a format that contains the following sections: Introduction, Equipment, Procedure, and Questions. HTML links are embedded to show graphics pictures of circuit diagrams, programs, etc. Figure 3 shows the procedure portion of Lab 6. A user can click on Procedure 1 to view how the circuit is configured to the A/D channel of the Opto22 Ethernet I/O module, as shown in Figure 4. Then, the user can click on Procedure 3 to obtain a temperature reading from the sensor and return this real-time temperature reading in an HTML document page as shown below.

\section{Analog Reading}

Current Temperature in Room 221 A: 74.5 F

Reading from LM 34, temperature sensor at point 25. 


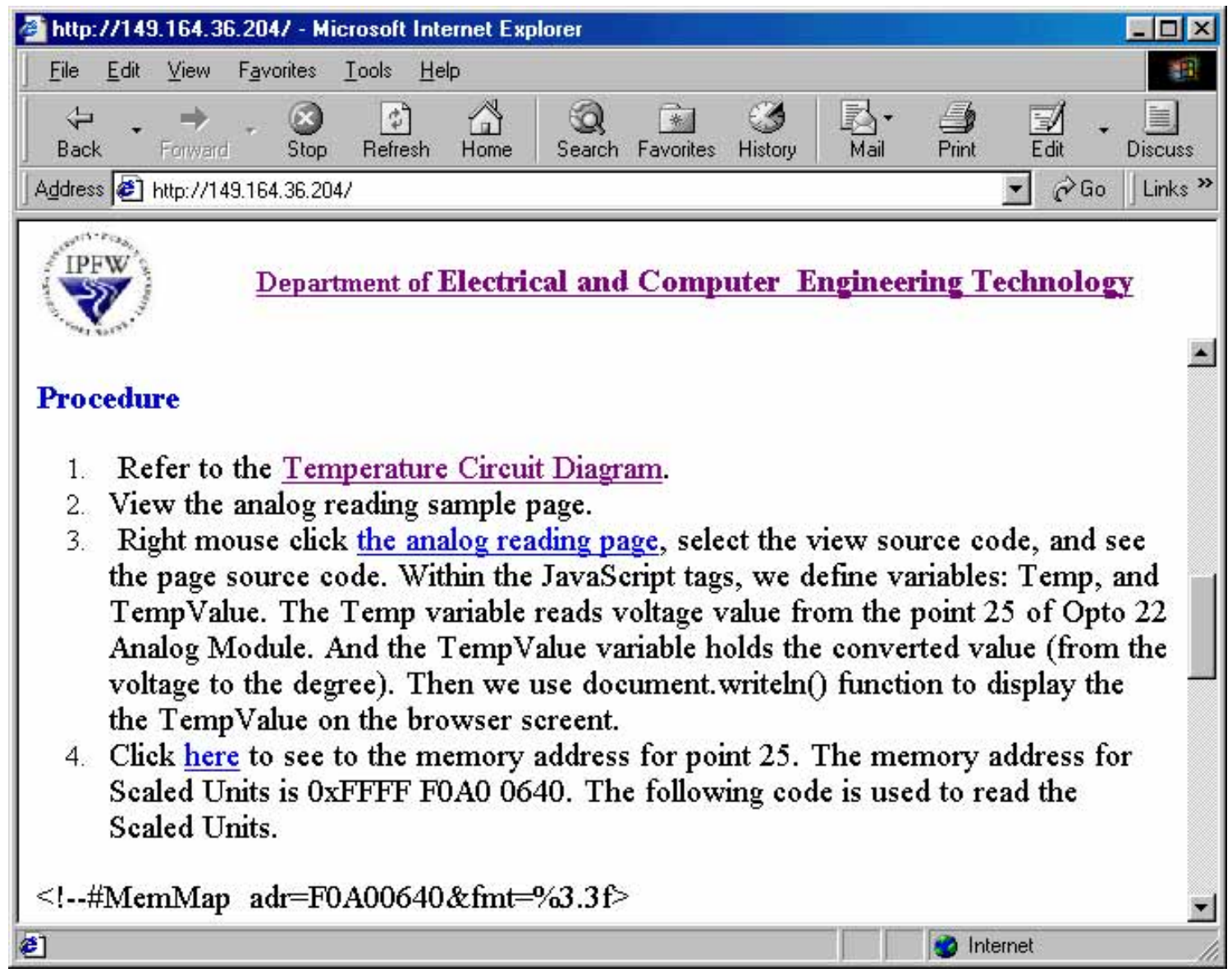

Figure 3. The Web Page of Lab 6

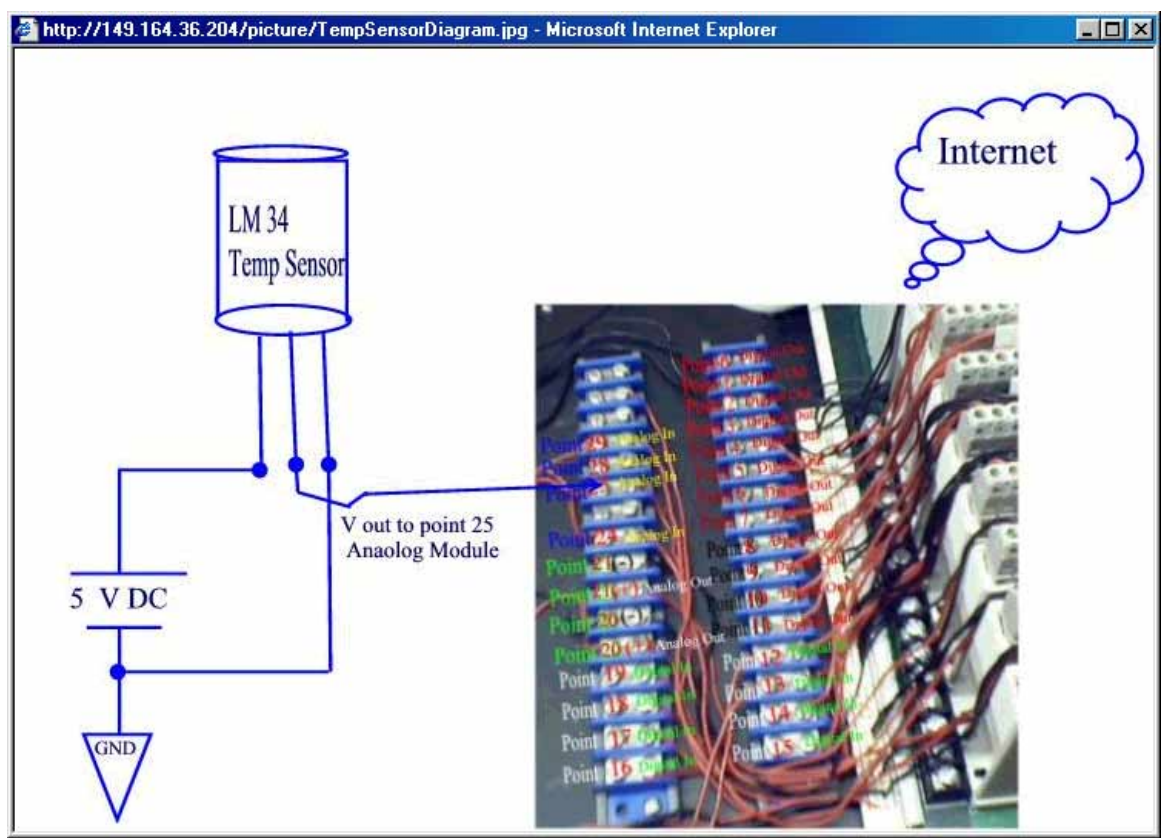

Figure 4. The Temperature Reading Circuit Diagram

Proceedings of the 2001 American Society of Engineering Education Annual Conference \& Exposition Copyright 2001, American Society for Engineering Education 


\section{Course Evaluation and Conclusion}

This course provided students with an opportunity to acquire new knowledge that might help their careers and prepare students for jobs in these challenging areas or in the areas of Internet, WWW, computers networking, Web programming, or electronics for measurement and control. This lab designed for asynchronous learning mode enhances IPFW's ability to serve nontraditional students and distance learners. It also reached students in other areas of the state. The students enrolled in this course for the Fall of 2000 semester were:

- 10 students enrolled in Electrical Engineering Technology (Networking Option) BS degree program

- 8 students enrolled in Electrical Engineering Technology BS degree program

- 3 students majoring in Computer science and/or Information system

- 3 students enrolled as distance learners currently studying in the Civil Engineering graduate program at Purdue University

- 1 practicing engineer with a need to broaden his knowledge in this area

In addition to homework, laboratories and tests, a course project (with 2 to 3 students forming a team) or comprehensive final exam is required for the completion of the course. The majority of students selected the term project option, while only two chose the final exam. Examples of final projects are:

- Web Enabled Factory Ventilation Control

- The Internet Counter Controller

- Web Database Access using JavaServlet (one ECET student and three CS students)

- Industrial Control Applications Using the Opto22 Factory Floor

- Home Security System (remote monitoring and control) using Ethernet I/O and Programmable Logic Controller

- Device and Appliance Control Over the Internet

- ASP Database Application: Data Time Clock

- Website Interface Controller and Database

- An Web Enabled Intelligent Traffic Monitoring System (Civil Engineering graduate students)

Student feedback is very encouraging. Examples of students comments are:

- ".. I just wanted to let you know how much I appreciated the CPET 499 Web Programming class. Being a student and working full time gives me a chance to see how useful this class can be in the real world. With the Internet changing in the work place, I see this type of class as a necessary for the Electrical and Computer Engineering Technology program. ... "

- " .., I want you to know that looking back over all the classes that I have ever taken I would rate this one as one of the best. It had very practical application for me since I was already involved in an OPTO 22 project. Even though I had never had html, C, C++, JavaScript, PERL or VB, my appetite was wetted enough that I 
would like to learn more about those languages. I would highly recommend this course for people like myself, who know programming fundamentals but need a jumpstart into the world of automation control using the Web. I would admit that at times the material was overwhelming but it made me dig all the harder. .."

\section{Bibliography}

1. Deitel, Deitel \& Nieto, Internet \& World Wide Web - How to Program, Prentice Hall, 2000

2. Paul I-Hai Lin, Tutorial On Java Computing for Distributed Applications over the Internet, IEEE IAS Annual Conference, Phoenix, Arizona, IEEE Industry Applications Society Publication, October 1999

3. Paul I-Hai Lin, Web Programming for Industrial Applications, www.etcs.ipfw.edu/ lin, Purdue University Fort Wayne Campus, Fall 2000

4. Manuel Aparicio, "Point \& Click Manufacturing on the Web, IEEE Internet Computing," September/October 1999, pp. 38-40

5. Fernando Zubillaga-Elorza and Charles Allen, "Virtual Instrument Toolkit: Rapid Prototyping on the Web," IEEE Internet Computing, September/October, pp. 41-48

6. Peter Deirauer and Bob Woolever, "Understanding Smart Devices," Industrial Computing, October 1998, pp. 47-50

7. Jini Network Technology Fact Sheet, www.sun.com/jini/factsheet, Sun Microsystem, January 25, 1999

8. Michael N. Huhns, "Networking Embedded Agents, IEEE Internet Computing, " Jan/Feb 1999, pp. 91-93

9. Behrouz Forouzan, Introduction to Data Communications and Networking, McGraw-Hill, 1998

10. Paul I-Hai Lin, "Distributed Control and Measurement via the Internet," Indiana Electronics manufacturing Association Annual Technical Conference, Indianapolis, Indiana, Nov. 4, 1999

11. Paul I-Hai Lin, Computer networking \& Data Communications: The Internet and Web Development technology Series, Indiana U-Purdue U Fort Wayne, October 2000

12. Paul I-Hai Lin, Building Web Applications with HTML: The Internet and Web Development technology SeriesI, Indiana U-Purdue U Fort Wayne, October 2000

13. Paul I-Hai Lin, Developing Advanced Web Applications Using JavaScript/Jscript: The Internet and Web Development technology Series, Indiana U-Purdue U Fort Wayne, October 2000

14. Paul I-Hai Lin, Programming with CGI/Perl: The Internet and Web Development technology Series Indiana U-Purdue U Fort Wayne, October 2000

\section{PAUL I-HAI LIN}

Lin is Associate Professor and Chair of Electrical and Computer Technology Department of Indiana University - Purdue University Fort Wayne. He has been with Purdue University since 1985. He is a registered Professional Engineer in Electrical Engineering at State of California and at the State of Indiana. Previously, he taught at Engineering and Technology Department of Dutchess Community College (NY) for three years (from 1982-1985), at Electrical Engineering Department of National Taipei Institute of Technology for two years, and worked in industry for 8 years. He was a Visiting Professor at National Taipei Science and Technology University (formally National Taipei Institute of Technology) during the spring of 1995. Lin is a Senior member of IEEE and was the Chairman of the Manufacturing Systems Development and Application Department of IEEE-Industry Applications Society from January 1998 to December 1999. Lin's current interests include distributed intelligent control of embedded real-time system, and sensors in industrial control applications. 


\section{HAL BROBERG}

Hal taught Electrical Engineering for 3 years at the U.S. Naval Academy and after retirement from the Marine Corps as a Lieutenant Colonel, chose to continue teaching. He received his PE license in Indiana in 1988 and his $\mathrm{PhD}$ in Engineering (EE) in 1993. His research area is servo systems and he has consulted and worked for ITT (Aerospace-Communications) on weather satellite servos for the past 9 years. He is currently an Associate Professor in the ECET department at Indiana Purdue University in Fort Wayne, IN, a senior member of IEEE and a program evaluator for IEEE with eleven TAC/ABET accreditation visits completed. 